sehütteln von Fruchtsäften mit Amylalkohol oder Aether zum Nachweise fremder Farbstoffe, Salicylsäure etc. Auch hier ist es von besonderem Vortheil, die Lösungsmittel für Farbstoffe, Salicylsäure u. dergl., durch ein besonderes Rohr ausfliessen lassen zu könneu, da besonders zuckerhaltiger Fruchtsaft stets am Rohr bängen bleibt und bei Verwendung eines gewöhnlichen Scheidetrichters die oben angefülırten Uebelstände besonders sich füblbar machen. Die Scheidetrichter mit Gestell werden in jeder beliebigen Grösse von der Firma C. L e ybold's Nachf. Cöln angefertigt.

Erlangen, Dezember 1897.

\title{
Ueber eine qualitative und quantitative Bestimmung von Weizenmehl im Roggenmehl.
}

(Mithoilung ans dem chemischen lnstitute in Mien.)

$$
\text { Vill }
$$

\section{S. Weinwarm.}

Die Unterscheidung verschiedener Mehlsorten erfolgt bekanntlich entweder durch die Grösse und Gestalt der Stärkekörner oder durch einzelne Verschiedenheiten in den sonstigen Gewebselementen. Früher wurde auf die erstere Art besonderer Werth gelegt, weil die Grösse und Gestalt der Stärkekörner in einigen Meblsorten wesenthich von einander verschieden ist und es nur einer geringen Uebung in der mikroskopischen Beobachtung bedarf, um konstatiren zu können, ob ein Roggen-, Mais- oder Hafermehl vorliegt. Einfach gestaltet sich noch immer die Aufgabe, ein reines Roggenmehl von einem reinen Weizenmehl zu unterscheiden, obwohl die Gestalt der Stärkekörner dieselbe ist, denn dic Grösse der Stärkekörner lässt hjer noch, ohne eine Messung vorzunehmen, einen Unterschied erkennen. Handelt es sich um die Prüfung eines Weizenmebles auf Zusatz von Roggenmehl, so unterliegt dies keinerlei Schwicrigkeit, weil schon die sternförmigen Risse der Roggenstärkekörner einen wesentlichen Anhaltspunkt bilden und ferner Bencke ${ }^{1}$ ) gefunden hat, dass das Roggenmell vorwiegend blaue, das Weizenmehl jedoch farblose, oder wenigstens keine blauen oder bläulichen Kleberzellen enthält.

Wesentlich schwieriger gestaltet sich jedoch der Nachwcis von Wcizenmehl in einem Roggenmehl. Um bier mit Sicherheit das Weizenmehl zu konstatiren, ist es nothwendig, Gewebsfragmente zur l'rüfung heranzuzichen. Wesentliche Verschiedenheiten in den Gewcbsfragmenten von Roggen - und Weizenmehl liegen nur in den Querzellen; denn während die letyteren an der Schmalseite Iückenlos aneinander schliessen und demnach frei sind von Intercellularräumen, bilden die Querzellen des Roggens an den Enden abgerundete,

1) T.undw. Yers...stat. 1884, 36, 337. 
porenfreie Formen, zwischen welchen Intercellularräume entstehen. Die Gegenwart von lückenlosen Querzellen war bisher der sicherste Beweis für das Vorhandensein von Weizenmehl. Diesen Nachweis zu erbringen, ist jedoch nicht in allen Fällen möglich, da besonders die feinsten Weizenmehle sehr wenig Kleientheile führen und dementsprechend nur selten derartige Querzellen zu finden sind. Die Gegenwart einer solchen Querzelle kann jedoch auch von einer zufälligen Verunreinigung herrühren. Ein anderes wichtiges Erkennungsmittel für Weizenmehl bilden die Haare, die, wenn sie auch kein solch sicheres Urtheil in der Unterscheidung von Weizen- und Roggenmehl zulassen wie die Querzellen, hingegen selbst in den feinsten Mehlen mit Leichtigkeit gefunden werden können. Im Allgemeinen ist anzunehmen, dass die Roggenhaare dünnwandig sind und weites Lumen zeigen, die Weizenhaare jedoch dickwandig sind und enges Lumen erkennen lassen, im Roggenmehl demnach grösstentheils weitlumige und im Weizenmehl englumige Haare gefunden werden. Neben den weitlumigen Haaren im Roggenmehl finden sich auch englumige, wie sie beim Weizen vorkommen, hingegen zeigen die Haare des Weizenmehles, mit Ausnahme der breiten bandförmigen Haare, nicht jenes weite Lumen, wie es bei den Roggenhaaren der Fall ist. Die Haare bilden demnach nicht jenes charakteristische Moment wie die Querzellen. Die von verschiedenen Forschern, wie Vogl ${ }^{1}$ ), Wittmack ${ }^{2}$ ) ausgeführten Messungen im Lumen und Wanddicke der Haare stimmen wohl untereinander nicht vollkommen überein, was übrigens auch nicht möglich ist; aus den angegebenen Zahlen beider Forscher ist jedoch ersichtlich, dass im Roggenmehl Haare vorkommen, welche an Wanddicke and Lumen denen des Weizenmehles ähnlich sind.

Ein weiteres Erkennungsmittel für die Anwesenheit von Weizenmehl im Roggenmehl bildet die Wittmack'sche ${ }^{3}$ ) Probe, nach welcher die Verkleisterungstemperatur beider Mehle zur Unterscheidung herangezogen wird. Diese Probe ist jedoch in dem von dem Forscher angegebenen Verfahren keineswegs so genau, um Beimengungen von 15\% Weizenmehl mit Sicherheit erkennen zu lassen, vorausgesetzt, dass nicht gleichzeitig mit dieser Probe die anderen Gewebsfragmente, wie Haare und Querzellen, zur Prüfung verwendet werden.

Nachdem jedoch Wittmack's Verkleisterungsprobe, sowie die Haare und Querzellen des Weizens, falls solche im Roggenmehl gefunden werden, keinerlei Anhaltspunkte über die Quantität des zugesetzten Weizenmehles liefern können, begnügte man sich, falls Zusätze konstatirt wurden, allgemein mit den Bezeichnungen „viel“, "wenig“, „beträchtlich" etc., welche Bezeichnungen jedoch, da eine blosse Schätzung zu Grunde lag, leicht zu Irrthümern Veranlassung gaben.

Eine auf dem Klebergehalte des Weizenmehles beruhende Methode hat für zollamtliche Revisionen $\mathrm{Bamihl}^{4}$ ) angegeben. Die Probe wurde neuerdings

1) Zeitschrift für Nahrungsmittel-Untersuchung, Hygiene und Waarenkunde 1897, 13, 220.

2) Wittmack's Anlejtung 31.

3) Wittmack's Anleitung 36 .

4) Poggendorf's Annalen 1852, 161. 
von Kleeberg ${ }^{1}$ ) wieder empfohlen, obwohl diesc Methode, wenn sie auch für den Zollbeamten am Platze war, für den Chemiker und Mikroskopiker nicht empfehlenswerth erscheint.

Quantitative Meblbestimmungen sind meines Wissens, abgesehen von der v, Weinzierl'schen mechanisch mikroskopischen Analyse ${ }^{2}$ ), nicht bekannt. Wahrscheinlich ist es, dass sich eine quantitative Meblbestimmung mit Erfolg nur auf Grund des Stärkegehalts, nicht jedoch auf andere Gewebstragmente gestützt, durchführen lässt.

Auch meine Methode stützt siclı auf die Stärkesubstanz und beruht auf folgendem Princip. Wird Roggenmehl bei $62^{1}, 63^{\circ} \mathrm{C}$. mit Wasser während einer Stunde digerirt, so sind fast alle Körner geruollen oder gelöst, sodass man hei mikroskopischer Beobachtung im Gesichtsfelde überhaupt gar keine oder nur sehr wenige Stärkekörner bemerkt, und diese zeigen grösstentheils keinen dunklen Kand. Wird jedoch Weizenmehl unter denselben Verhäitnissen digerirt, so sind wohl auch diese Stärkekörner gequollen, aber es zeigen fast alle Körner dunklen Rand. Man kann nun aus der Zahl der noch dunklen Rand zeigenden Körner auf die Quantität des zugesetzten Mehles einen Schluss ziehen. Die Probe wird in folgender Weise ausgeführt: $2 \mathrm{~g}$ Mehl werden mit $200 \mathrm{ccm}$ Wasser während einer Stunde bei $621 / 3-63^{\circ} \mathrm{C}$. digerirt. Man nimmt mehrere möglichst gleich grosse Tropten aus dem Gemenge, breitet dieselben gleichmässig auf dem Objektträger aus und zählt an 20 verschiedencn Stellen des Deckglases jene Stärkekörner, welche dunklen Rand zeigen. Es crgiebt sich bei Verwendung desselben Objektivs ${ }^{3}$ ), bei Beobachtung von 5 Tropfen und unter Beibehaltung gleicher Verhältnisse folgendes Resultat:

$\begin{array}{ccc}\text { Roggenmohl } & \text { Wïinmehl } & \text { Körner mit dunklem liand } \\ 100 \% & 0_{i 0}^{0:} & 25 \\ 95- & 5- & 38 \\ 90- & 10- & 63 \\ 85- & 15- & 93 \\ 80- & 20- & 136 \\ 75- & 25- & 160\end{array}$

Es zeigt sich bei dieser Methode, dass jene Stärkekörner, welche nach dem Digeriren noch einzeln oder in Gruppen in den Endospermzellen enthalten sind, nicht gequollen sind, sondern nur Risse zeigen und eine den Maisstärkekörnern ähnliche Form angenommen baben; diese Körner, sowic sonstige kleine Körner, werden nicht in Berücksichtigung gezogen.

Bei der Lntersuchung von zwei Tropfen ergeben sich oft gleichviel Körner mit dunklem Rand, manchmal jedoch zeigen sich, wie nicht anders erwartet werden kann, wesentliche Differenzen. Untersucht man jedoch, wie dies bei quantitativen Bestimmungen selbstverständlich ist, mehrere Tropfen, so ergeben

1) Chem. Ztg. 1892, 16, 1071.

2) Zeitschrift für Nahrungsmittel-Untersuchung, Hygiene und Waarenkunde 1887, 1, 118.

3) Reichert No. 6. 
sich im Durchschnitt gut übereinstimmende Resultate, welche für die Praxis mehr als hinlänglich genau sind.

Bei qualitativen Prüfungen, zur Beantwortung der Frage also, ob überhaupt Weizenmehl vorhanden ist, genügt schon die Untersuchung von zwei Tropfen. Mangels einer quantitativen Methode war die Prüfung bisher stets nur qualitativ, in Zukunft kann jedoch die Frage, ob Roggenmehl $5 \%$ oder mehr Weizenmehl enthält, mit vollster Sicherheit beantwortet werden. Nach diesem Verfahren gelingt somit der Nachweis des Weizenmehles nur durch Prüfung der Stärke, ohne Rücksicht auf andere Gewebsfragmente nehmen zu müssen, was speciell bei feineren Mehlsorten von wesentlichem Vortheil ist.

Zur Prüfung wurden absichtlich Futtermehle verwendet, um den störenden Einfluss der Kleientheile, sowie sonstiger Verunreinigungen berücksichtigen zu können, da bei der Herstellung dieser Mehle oft nicht jene Sorgfalt verwendet wird, wie bei den besseren Mehlsorten. Diese Methode lässt sich wahrscheinlich auch bei anderen Mehlarten mit Erfolg verwenden, und behalte ich mir diesbezügliche weitere Arbeiten auf diesem Gebiete vor.

\section{Beiträge zur Kenntniss des Kaffees und der Kaffeesurrogate.}

\section{Die Methoden der Kaffeegerbsänrebestimmang.}

$$
\text { Iin }
$$

\section{Heinrich Trillich und Wr. H. (Göckel.}

Wie aus den von Bibra') bethätigten Zusammenstellungen älterer Untersuchungen über Kaffee hervorgeht, bestanden schon damals ganz wesentliche Abweichungen in den Ergebnissen.

Abgeseben von den Forschungen nach dem innern Aufbau der Kaffeegerbsäurc ist dieselbe lange Zeit kein Gegenstand des Studiums, insbesondere nicht der Nahrungsmittclchemiker gewescn, sodass selbst grössere Werke über Methoden der Kaffeegerbsäurebestimbiung keine $\Lambda$ ngaben enthalten.

König führt eine von Bell'2) angegebene Methode an, Wile $\mathrm{y}^{3}$ ) eine solche von W. H. Krug. Neuerdings veröfientlichten Herfeldt und Stutzert) Beubachtungen gelegentlich Versuchen, die Kaffecgerbsäurc zu bestimmen, dic aber zu keinen weiteren Versuchen aufmuntern können.

Von den Forschungen Kuntz-Krause's') über die Struktur der Kaffeegerbsäure lassen sich vielleicht für die Analyse zweckdienlicbe Aufschlüsse erwarten.

1) Der Kaffee und seine Surrogate. Mün'hen 1856.

2) Analyse und Verfälschung der Niahung̣smiltel. v. Mirus: 188?. 18.

3) Foods \& Foods Adulterants, 7, 1892.11 :thington.

4) Zeitschr. angew. Chem. 1895, 469.

5) Ber. deutsch. chem. Ges. 1897, 30, 1617. 